\title{
In Situ Optical Microscopy of the Electrochemical Intercalation of Lithium into Single Crystal Graphite
}

\author{
Jared J. Lodico ${ }^{1}$, Mark Woodall ${ }^{1}$, Ho L Chan $^{1}$, William A. Hubbard ${ }^{1}$, and B. C. Regan ${ }^{1}$ \\ 1. Department of Physics \& Astronomy and California NanoSystems Institute, University of California, \\ Los Angeles, California 90095 USA
}

The lithium ion battery (LIB) is an energy storage technology that is widely used in consumer electronics [1]. Though many cathode materials have been successfully used in various types of LIB, graphite is still the preferred anode material [2]. A graphite intercalation compound (GIC) is formed during the battery's charging process as lithium ions are inserted, or intercalated, between the individual graphene layers. Even though GICs have been researched extensively [3], the electrode/electrolyte interface and the kinetics of the intercalant during the intercalation process are not fully understood $[1,4]$. We use optical microscopy to observe the intercalation and deintercalation of lithium ions and quantify color changes, which are directly related to the host's structural evolution, within micron-scale, single-crystal graphite flakes.

We mechanically exfoliated bulk natural graphite (NGS Naturagraphit GmBH) and transferred individual crystalline flakes with wet processing techniques to a nickel electrode on a glass slide. To minimize its contribution to the electrical transport data, the nickel electrode was almost entirely covered with $\mathrm{SiO}_{2}$ deposited via e-beam evaporation. The electrolyte, a $1 \mathrm{M}$ lithium perchlorate $\left(\mathrm{LiCLO}_{4}\right)$ solution in ethylene carbonate (EC):dimethyl carbonate (DMC) (1:1 ratio by volume), was then added. Finally, the Ni/graphite/electrolyte stack was covered with a cover slip (thickness $\sim 150 \mu \mathrm{m}$ ) to form an optically-transparent fluid cell. Lithium foil was used for both the reference (RE) and counter (CE) electrodes. All in situ optical experiments were conducted in an argon atmosphere at room temperature.

Using a Gamry Reference 600 potentiostat, a graphite flake's potential was ramped at $1 \mathrm{mV} / \mathrm{s}$ from its open circuit potential (OCP), $3.05 \mathrm{~V}$, to an intercalating voltage of $5 \mathrm{mV}$. The potential was held at $5 \mathrm{mV}$ for 3.5 hours to further intercalate the flake before returning to the OCP at $0.5 \mathrm{mV} / \mathrm{s}$. Figure 1 shows a time series of optical images (a) of an initially pristine graphite flake $(0.15 \mu \mathrm{m} \times 94 \mu \mathrm{m}$ $\times 172 \mu \mathrm{m}$ ) subject to this regimen, and the corresponding transport data (b). As the graphite flake experienced its first electrochemical intercalation and deintercalation cycle, it changed color based on the composition of the GIC.

GICs are characterized by their stage number: stage $n$ has $n$ layers of graphene between each layer of intercalant. The colors of the unintercalated, dilute stage 2, stage 2, and stage 1 Li-GICs are grey, blue, red, and gold respectively [5]. Starting from its initial ( $t=0 \mathrm{~min})$, pristine state, the flake in Figure 1 became predominantly dilute stage 2 by $t=62 \mathrm{~min}$, as indicated by the color change from grey to blue. As the potential was held at $5 \mathrm{mV}$ the perimeter of the flake first turned gold. Red, then gold intercalation waves propagated inwards from the perimeter of the graphite toward the center until the majority of the flake became gold colored $(t=260 \mathrm{~min})$. After the potential began to ramp back toward the OCP, the graphite deintercalated. The color changes associated with deintercalation happened more quickly than those of intercalation and were also accompanied by a distinct current peak in the electrical transport data (Figure 1b). After the intercalant was expelled at $t=363$ min the graphite was left with 
linear defects spanning the entire flake. The combination of electrochemical transport measurements and in situ imaging of single microcrystals of graphite promises to elucidate the dynamics of intercalation and deintercalation.

References:

[1] M. Gauthier et al, J. Phys. Chem. Lett. 6 (2015), p. 4653-4672.

[2] D. Olson et al, U.S. Geological Survey, (2013), p. 1-11.

[3] M. S. Dresselhaus and G. Dresselhaus, Adv. Phys. 30 (1981), p. 139-326.

[4] A. Funabiki et al, J. Electrochem. Soc. 146 (1999), p. 2443-2448.

[5] S. J. Harris et al, Chem. Phys. Lett. 485 (2010), p. 265-274.

[6] This work was supported by National Science Foundation (NSF) award DMR-1611036, by NSF STC award DMR-1548924, and by FAME, one of six centers of STARnet, a Semiconductor Research Corporation program sponsored by MARCO and DARPA.

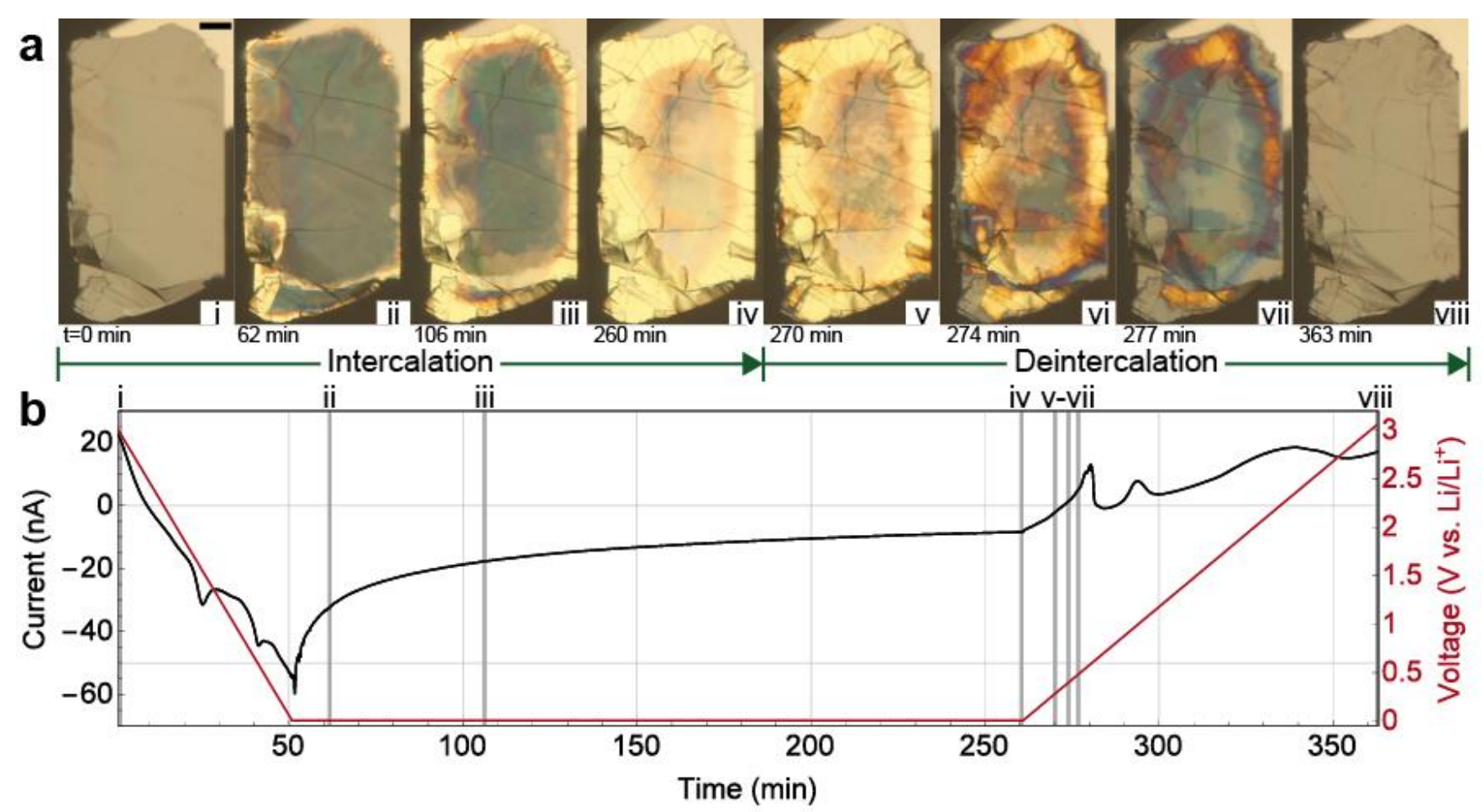

Figure 1. (a) A time series of optical images during the first intercalation/deintercalation cycle of a graphite flake, which is electrically connected to a nickel electrode (top right). Both the reference and counter electrodes are lithium foil (outside the field of view). The scale bar is $20 \mu \mathrm{m}$. (b) The electrical transport data acquired simultaneously with the optical images. The red (black) curve is the voltage (current) versus time. Grey lines in (b) indicate when the images in (a) were acquired. 